\title{
Exactly solvable Kitaev model in three dimensions
}

\author{
Saptarshi Mandal ${ }^{1}$ and Naveen Surendran ${ }^{2}$ \\ 1 The Institute of Mathematical Sciences, C.I.T Campus, Chennai 600113, Indi柾 \\ ${ }^{2}$ The Abdus Salam International Centre for Theoretical Physics, Strada Costiera 11, 34014 Trieste, Italy
}

(Dated: November 4, 2018)

\begin{abstract}
We introduce a spin- $\frac{1}{2}$ model in three dimensions which is a generalization of the well-known Kitaev model on a honeycomb lattice. Following Kitaev, we solve the model exactly by mapping it to a theory of non-interacting fermions in the background of a static $\mathbb{Z}_{2}$ gauge field. The phase diagram consists of a gapped phase and a gapless one, similar to the two-dimensional case. Interestingly, unlike in the two-dimensional model, in the gapless phase the gap vanishes on a contour in the k space. Furthermore, we show that the flux excitations of the gauge field, due to some local constraints, form loop like structures; such loops exist on a lattice formed by the plaquettes in the original lattice and is topologically equivalent to the pyrochlore lattice. Finally, we derive a low-energy effective Hamiltonian that can be used to study the properties of the excitations in the gapped phase.
\end{abstract}

PACS numbers: 75.10.Jm,03.67.Pp,71.10.Pm

\section{INTRODUCTION}

The study of topological phases has been actively pursued in condensed-matter systems for some years. This has resulted in the emergence of a new paradigm in the theory of quantum phase transitions: certain phase transitions cannot be described in terms of local order parameters associated with spontaneously broken symmetries; instead, the phases in such transitions are characterized by topological order 1 The most famous example of topological order in a quantum system is in the phenomenon of fractional quantum Hall effect.2,3,4 Other examples - experimental and theoretical -include quantum spin liquids,,$\frac{5,6,7,8}{4}$ quantum dimer models, $, 9,10,11$ quantum loop models, 12 etc.

Recently, it has been proposed that topological phases can be used to do quantum computation.13.14 The main obstacle in the realization of quantum memory - the basic ingredient of a quantum computer - is decoherence: it is difficult to prepare states that are robust to external noise. Kitaev $\stackrel{15}{ }$ suggested that topologically ordered states can be used to overcome this problem. He illustrated these ideas in a spin- $\frac{1}{2}$ model on a hexagonal lattice which, quite remarkably, can be solved exactly. The ground state of the Kitaev model has two phases: in one phase the elementary excitations have a gap in the spectrum and are Abelian anyons; the second phase is gapless in the absence of an external magnetic field, but develops a gap when the field is switched on, and then the excitations are non-Abelian anyons. In topological quantum computation, braiding of non-Abelian anyons is essential for the realization of universal quantum gates 16 Regarding the feasibility of a physical realization of the Kitaev model, there has been a proposal to realize it on an optical lattice $, \underline{17}, 18$

Apart from its potential application in quantum computation, the Kitaev model is an interesting many-body system by itself. First, exact solutions are rare in dimensions higher than one, and second, the model provides a relatively simple platform - the Hamiltonian involves only two-body interactions - to study concepts such as topological order and fractional excitations. Thus, not surprisingly, the various many-body aspects of the model have been thoroughly investigated, $19,20,21,22,23,24$ There have also been some generalizations to other twodimensional (2D) lattices ${ }^{25,26}$ It is then worthwhile to find generalizations of the model in higher dimensions. In this paper, we introduce and study a three-dimensional (3D) version of the Kitaev model. Three-dimensional models exhibiting topological order have been studied previously. $27,28,29,30$

An exact solution of the Kitaev model is possible due to the existence of a macroscopic number of locally conserved quantities: this facilitates the mapping of the model to a quadratic Hamiltonian of Majorana fermions hopping in the background of a static $\mathbb{Z}_{2}$ gauge field. The three-dimensional model we construct also has the above feature, which renders it exactly solvable. It has a gapped phase and a gapless one, just as in 2D, and although the phase boundaries are identical to the latter, the nature of certain excitations is quite different. In the three-dimensional model, the excitations of the gauge field are localized on "loops". This gives rise to the possibility that, as yet unverified in our model, such excitations can obey nontrivial statistics, since double exchange in loops - unlike points - is topologically nontrivial in three dimensions (for example, see Ref. 29).

The paper is organized as follows: in Sec. II we define the spin Hamiltonian and then rewrite it using a Majorana fermion representation of spin $\frac{1}{2}$. In Sec. III the low-energy spectrum for fermionic excitations is derived while Sec. IV] discusses the excitations of the gauge field. We then derive the low-energy effective Hamiltonian in the large $J_{z}$ limit in Sec. $\mathrm{V}$ ] and end with a discussion of our results in the last section. 


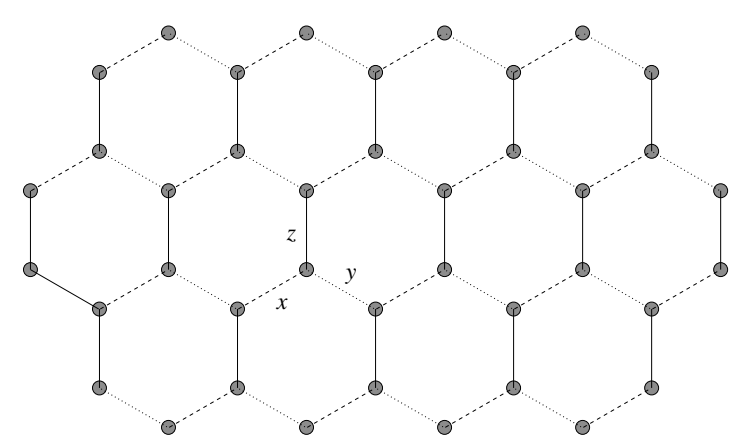

FIG. 1: The honeycomb lattice: the three types of links are labeled $x, y$, and $z$.

\section{HAMILTONIAN}

Before presenting the three dimensional model, let us briefly describe the Kitaev model in 2D. It is a system of spin- $\frac{1}{2}$ degrees of freedom located at the vertices of a honeycomb lattice. In the honeycomb lattice, there are three types of links which, distinguished by their orientations, are labeled $x, y$ and $z$ (see Fig. 11). The Kitaev Hamiltonian is

$$
H=-J_{x} \sum_{<i, j>_{x}} \sigma_{i}^{x} \sigma_{j}^{x}-J_{y} \sum_{<i, j>_{y}} \sigma_{i}^{y} \sigma_{j}^{y}-J_{z} \sum_{<i, j>_{z}} \sigma_{i}^{z} \sigma_{j}^{z},
$$

where $\sigma^{a}$ 's are the Pauli matrices, and $\langle i, j\rangle_{a}$ indicates that $i$ and $j$ belong to a link of $a$-type.

For our purposes, the key points to note about the Hamiltonian in Eq. (11) are that for any spin, only one of the components couples to a particular neighboring spin, and the component with non-zero coupling strength is different for each of the three neighbors. As we will see later, this leads to the existence of a set of mutually commuting conserved plaquette operators, which in turn makes the problem exactly solvable.

We note two features of the honeycomb lattice that are pertinent to the construction of the 3D lattice: (1) The coordination number of the lattice is 3. (2) The three types of links $x, y$ and $z$ are distributed in such a way that two links of the same type do not touch each other.

\section{A. Lattice}

To facilitate visualization, we will first describe how to obtain the 3D lattice starting from the familiar cubic lattice. Let $i, j, k \in \mathbb{Z}$ be the $x, y$ and $z$ coordinates of the latter. The new lattice is obtained by removing those sites that satisfy one of the following conditions: (1) $k=0 \bmod 4$ and $i=0 \bmod 2,(2) k=1 \bmod 4$ and $j=0 \bmod 2,(3) k=2 \bmod 4$ and $i=1 \bmod 2$, and (4) $k=3 \bmod 4$ and $j=1 \bmod 2$.

This amounts to depleting the cubic lattice by half, and the resultant lattice has coordination number 3 (see Fig. 21). We note that: (i) The $x-y$ planes alternately consist of disconnected rows or disconnected columns. (ii) As one goes along a particular row (column), at each site there is a link whose direction alternates between positive and negative $z$ axes. That is, there is a criss-crossing structure between adjacent planes which ensures that the lattice is truly three dimensional - despite a coordination number of 3-and not a set of mutually disconnected twodimensional surfaces.



FIG. 2: The 3D lattice: the four sites inside the loop (marked 1-4) constitute a unit cell; $\mathbf{a}_{1}, \mathbf{a}_{2}$, and $\mathbf{a}_{3}$ are the basis vectors. Plaquette $p$ consists of sites marked 1-10.

To parametrize the lattice sites, we first note that the unit cell contains four sites. The position vector of a unit cell is given by

$$
\begin{aligned}
\mathbf{R} & =m \mathbf{a}_{1}+n \mathbf{a}_{2}+p \mathbf{a}_{3}, \quad m, n, p \in \mathbb{Z}, \\
\mathbf{a}_{1} & =2 \hat{\mathbf{x}}, \quad \mathbf{a}_{2}=2 \hat{\mathbf{y}}, \quad \mathbf{a}_{3}=\hat{\mathbf{x}}+\hat{\mathbf{y}}+2 \hat{\mathbf{z}},
\end{aligned}
$$

where $\hat{\mathbf{x}}, \hat{\mathbf{y}}$, and $\hat{\mathbf{z}}$ are unit vectors along $x, y$ and $z$ directions, respectively. The four sites within a unit cell are at

$$
\begin{aligned}
\mathbf{r}_{1}=\mathbf{R}-\frac{\hat{\mathbf{y}}}{2}-\hat{\mathbf{z}}, & \mathbf{r}_{2}=\mathbf{R}-\frac{\hat{\mathbf{y}}}{2}, \\
\mathbf{r}_{3}=\mathbf{R}+\frac{\hat{\mathbf{y}}}{2}, & \mathbf{r}_{4}=\mathbf{R}+\frac{\hat{\mathbf{y}}}{2}+\hat{\mathbf{z}}
\end{aligned}
$$

To define a Kitaev-type Hamiltonian, we need one more ingredient, viz, the labeling of links. To this end, we alternately assign $x$ and $y$ labels to the links in each of the rows and columns that lie on the $x-y$ plane; the remaining links, the ones along the $z$ axis, are labeled $z$. [The ambiguity in the assignment of $x$ and $y$ labels within each row (column) is resolved by demanding periodicity.] This way of labeling ensures that the three links emanating 
from each site have different labels. Now the definition of the Hamiltonian in Eq. (11) can be applied to the three- dimensional lattice we have constructed. Explicitly,

$$
\begin{gathered}
H=\sum_{\mathbf{R}}\left[-J_{x} \sigma_{1}^{x}(\mathbf{R}) \sigma_{4}^{x}\left(\mathbf{R}-\mathbf{a}_{3}\right)-J_{y} \sigma_{1}^{y}(\mathbf{R}) \sigma_{4}^{y}\left(\mathbf{R}+\mathbf{a}_{1}-\mathbf{a}_{3}\right)-J_{x} \sigma_{2}^{x}(\mathbf{R}) \sigma_{3}^{x}(\mathbf{R})\right. \\
\left.-J_{y} \sigma_{3}^{y}(\mathbf{R}) \sigma_{2}^{y}\left(\mathbf{R}+\mathbf{a}_{2}\right)-J_{z} \sigma_{1}^{z}(\mathbf{R}) \sigma_{2}^{z}(\mathbf{R})-J_{z} \sigma_{3}^{z}(\mathbf{R}) \sigma_{4}^{z}(\mathbf{R})\right]
\end{gathered}
$$

For simplicity of notation, we will continue to use the formal expression for $H$ in Eq. (10), where sites are referred to by a single lower-case index, and revert to the explicit form in Eq. (5) only when the calculation demands it.

\section{B. Conserved quantities}

Let $l=\left(j_{1}, j_{2}, \ldots, j_{n}\right)$ be a sequence of lattice sites such that $j_{m}$ and $j_{m+1}$ are neighbors for $m=1,2, \ldots n$ with $j_{n+1} \equiv j_{1}$. Then $l$ represents a loop. Equivalently, the links formed among $j_{m}$ also uniquely determine the loop. Let

$$
W_{l}=\prod_{j_{m} \in l} \sigma_{j}^{a_{j_{m}}},
$$

where $a_{j_{m}}$ is the label of the link that connects $j_{m}$ to its neighbor which is not in $l$. It is easy to see that

$$
\left[W_{l}, H\right]=0,\left[W_{l}, W_{l^{\prime}}\right]=0, \forall l, l^{\prime} .
$$

However, not all such operators are independent. To see this, we define a geometrical operation of combining two loops as follows: the combination of loops $l_{1}$ and $l_{2}$ is the loop $l_{12}$ formed by all the links in $l_{1}$ and $l_{2}$ except those that are common to both. Then, it is easy to see that $W_{l_{1}} W_{l_{2}}= \pm W_{l_{12}}$. This means that for a lattice with open boundary conditions, any loop operator can be written as a product of those defined on the plaquettes - the elementary loops which cannot be obtained by combining smaller loops. For example, in Fig. 2. $W_{p}$ corresponding to the plaquette $p$, consisting of the sites marked $1,2, \ldots, 10$, is

$$
W_{p}=\sigma_{1}^{x} \sigma_{2}^{y} \sigma_{3}^{y} \sigma_{4}^{y} \sigma_{5}^{z} \sigma_{6}^{x} \sigma_{7}^{y} \sigma_{8}^{y} \sigma_{9}^{y} \sigma_{10}^{z} .
$$

There are further constraints among $W_{p}$ corresponding to different plaquettes, and we will discuss them in Sec. [V] where the excitations are studied.

\section{Hamiltonian in Majorana fermion representation}

To solve the Hamiltonian, following Kitaev $\stackrel{15}{,}$ we use a representation of Pauli matrices in terms of Majorana fermions. At each site $j$, we introduce four Majorana operators $b_{j}^{x}, b_{j}^{y}, b_{j}^{z}$ and $c_{j}$, which satisfy the following relations.

$$
\begin{aligned}
b_{j}^{\alpha \dagger}=b_{j}^{\alpha}, & c_{j}^{\dagger}=c_{j}, \\
\left\{b_{j}^{\alpha}, b_{k}^{\beta}\right\}=2 \delta_{j k} \delta_{\alpha \beta}, & \left\{c_{j}, c_{k}\right\}=2 \delta_{j k}, \\
\left\{b_{j}^{\alpha}, c_{l}\right\}= & 0 .
\end{aligned}
$$

The Majorana fermions act on a Fock space which is four dimensional, whereas the spin Hilbert space has two dimensions. We define the physical space as consisting of those states which satisfy the constraint

$$
D_{j}|\xi\rangle=|\xi\rangle, \quad \forall j \quad \text { where } D_{j}=b_{j}^{x} b_{j}^{y} b_{j}^{z} c_{j} .
$$

$D_{j}^{2}=1$; thus, $\left(1, D_{j}\right)$ form the elements of a $\mathbb{Z}_{2}$ gauge group. The spin operators are defined as

$$
\sigma_{j}^{\alpha}=i b_{j}^{\alpha} c_{j}
$$

When restricted to the physical space, the above operators satisfy the standard spin- $\frac{1}{2}$ algebra. The projector to the physical space is given by

$$
P_{j}=\frac{1+D_{j}}{2} .
$$

All the states related by a gauge transformation project on to the same physical state.

Starting from a generic spin model, $H\left\{\sigma_{j}^{\alpha}\right\}$, we can obtain a fermionic Hamiltonian, $\widetilde{H}\left\{b_{j}^{\alpha}, c_{j}\right\}$, by using Eq. (11). Since $\left[\widetilde{H}, P_{j}\right]=0 \forall j$, the eigenstates of $H$ can be obtained from those of $\widetilde{H}$ by projecting the latter to the physical space.

Substituting Eq. (11) in Eq. (1), we obtain

$$
\begin{aligned}
& \widetilde{H}=\frac{i}{2} \sum_{j, k} \hat{A}_{j k} c_{j} c_{k}, \\
& \hat{A}_{j k}=\left\{\begin{array}{cl}
J_{\alpha_{j k}} \hat{u}_{j k} & \text { if } j \text { and } k \text { are linked, } \\
0 & \text { otherwise, }
\end{array}\right. \\
& \hat{u}_{j k}=i b_{j}^{\alpha_{j k}} b_{k}^{\alpha_{j k}} \text {, }
\end{aligned}
$$

where $\alpha_{j k}$ is the type of the link between $j$ and $k$. We note that $\hat{u}_{j k}=-\hat{u}_{k j}$, and in the sum the links are treated as directed and therefore counted twice. We use 
a hat to emphasize that $\hat{u}_{j k}$ is an operator; $u_{j k}$ is the corresponding eigenvalue and takes values \pm 1 . Furthermore,

$$
\left[\widetilde{H}, \hat{u}_{j k}\right]=0,\left[\hat{u}_{j k}, \hat{u}_{l m}\right]=0 \forall<j, k>,<l, m>.
$$

Therefore, the Hilbert space breaks up into various sectors, each corresponding to a particular set $\left\{u_{j k}\right\}$; the matrix elements of $\widetilde{H}$ between states belonging to different sectors are zero. The Hamiltonian in a given sector is obtained by replacing the operators $\hat{u}_{j k}$ with their corresponding eigenvalues, $u_{j k}$ :

$$
\widetilde{H}_{u}=\frac{i}{2} \sum_{j, k} A_{j k} c_{j} c_{k},
$$

where $A_{j k}$ is obtained from Eq. (14) by substituting $\hat{u}_{j k}$ with $u_{j k}$. The gauge-invariant and hence physical conserved quantities are

$$
\widetilde{W}_{p}=\prod_{m=1}^{10} \hat{u}_{j_{m} j_{m+1}},
$$

where $p=\left(j_{1}, j_{2}, \ldots, j_{10}\right)$, as before, is a plaquette. $\widetilde{W}_{l}$ is related to $W_{l}$ in Eq. (6) as follows:

$$
W_{l}=\mathcal{P}_{l} \widetilde{W}_{l} \mathcal{P}_{l}, \quad \mathcal{P}_{l}=\prod_{j \in l} P_{j}
$$

From now on we will simplify the notation, following Kitaev 15 by not making distinction between operators in the physical and extended Hilbert spaces, i.e., we will drop tilde from all the operators acting in the latter.

\section{GROUND STATE AND SPECTRUM}

Next question to be addressed is: Which sector of $\left\{u_{i j}\right\}$ does the ground state belong to? The problem of free fermions hopping on a $d$-dimensional hypercubic lattice with hopping amplitude $\left|t_{i j}\right| e^{i \theta_{i j}}$ between nearestneighbor sites $i$ and $j$ was studied by Lieb. 31 Moreover it was shown that, if $\left|t_{i j}\right|$ is reflection symmetric about certain planes that does not contain any sites, then the ground-state energy is the lowest when the flux of the phase along the plaquettes, $\Phi \equiv \sum_{<i j>} \theta i j$, equals $\pi$ if the length of the loop is $0 \bmod 4$, and zero if the length is $2 \bmod 4$. Unfortunately, Lieb's result cannot be directly applied to our case because our lattice does not have the required reflection symmetry.

We numerically studied the ground-state energy of the Hamiltonian in Eq. (17) for lattices containing up to 864 sites using periodic boundary conditions. The groundstate energy in the sector with uniform flux $\left(W_{p}=\right.$ $+1, \forall p)$ was compared with that of other selectively chosen flux configurations. As will be discussed in Sec. IV. due to some local constraints, the plaquettes that are excited themselves form loops in an embedded lattice. The smallest such loop consists of six plaquettes. We considered excitations of loops of varying length and found the energy increasing with increasing length. We also looked at excitations of multiple loops. In every case considered, we found that the energy of the lowest energy state is greater than that of the $W_{p}=+1$ uniform flux state. In Sec. V] we will get further confirmation of this result, at least in the large $J_{z}$ limit, through a perturbative analysis. Therefore it is reasonable to assume that the ground state belongs to this sector.

$u_{j k}=1$ for all links is the obvious choice among the configurations of link variables that gives $W_{p}=+1, \forall p$. Of course, any configuration related to this one by a gauge transformation will also satisfy the above condition on $W_{p}$ 's. With this choice, the Hamiltonian in its explicit form becomes

$$
\begin{gathered}
H=i \sum_{\mathbf{R}}\left[J_{x} c_{1}(\mathbf{R}) c_{4}\left(\mathbf{R}-\mathbf{a}_{3}\right)+J_{y} c_{1}(\mathbf{R}) c_{4}\left(\mathbf{R}+\mathbf{a}_{1}-\mathbf{a}_{3}\right)+J_{x} c_{2}(\mathbf{R}) c_{3}(\mathbf{R})\right. \\
\left.+J_{y} c_{3}(\mathbf{R}) c_{2}\left(\mathbf{R}+\mathbf{a}_{2}\right)+J_{z} c_{1}(\mathbf{R}) c_{2}(\mathbf{R})+J_{z} c_{3}(\mathbf{R}) c_{4}(\mathbf{R})\right]
\end{gathered}
$$

where $\mathbf{r}$ and $\mathbf{a}_{i}$ are given in Eqs. (2) and (3). To diagonalize the Hamiltonian, we next do a Fourier transform.

$$
c_{\mu}(\mathbf{r})=\int_{-\pi}^{\pi} \frac{d k_{1}}{2 \pi} \int_{-\pi}^{\pi} \frac{d k_{2}}{2 \pi} \int_{-\pi}^{\pi} \frac{d k_{3}}{2 \pi} e^{-i \mathbf{k} \cdot \mathbf{r}} c_{\mu \alpha}(\mathbf{k}),
$$

with $\mu=1,2,3,4$ and where

$$
\mathbf{k}=k_{1} \mathbf{b}_{1}+k_{2} \mathbf{b}_{2}+k_{3} \mathbf{b}_{3},
$$

and

$$
\mathbf{b}_{1}=\frac{(2 \hat{\mathbf{x}}-\hat{\mathbf{z}})}{4}, \quad \mathbf{b}_{2}=\frac{(2 \hat{\mathbf{y}}-\hat{\mathbf{z}})}{4}, \quad \mathbf{b}_{3}=\frac{\hat{\mathbf{z}}}{2} .
$$

Using the property, $c_{\mu}(-\mathbf{k})=c_{\mu}^{\dagger}(\mathbf{k})$, the Hamiltonian becomes, 


$$
H=\int_{-\pi}^{\pi} \frac{d k_{1}}{2 \pi} \int_{-\pi}^{\pi} \frac{d k_{2}}{2 \pi} \int_{-\pi}^{\pi} \frac{d k_{3}}{2 \pi}\left[\frac{i}{2}\left\{e^{i k_{3}} \delta_{k_{1}} c_{1}^{\dagger}(\mathbf{k}) c_{4}(\mathbf{k})+\delta_{k_{2}} c_{3}^{\dagger}(\mathbf{k}) c_{2}(\mathbf{k})+J_{z} c_{1}^{\dagger}(\mathbf{k}) c_{2}(\mathbf{k})\right\}+\text { h.c. }\right],
$$

where $\delta_{k_{i}}=J_{x}+e^{-i k_{i}} J_{y}$, for $i=1,2$. Furthermore, we define $\Delta_{\mathbf{k}}=\left(\left|\delta_{k_{1}}\right|^{2}+\left|\delta_{k_{1}}\right|^{2}+2 J_{z}^{2}\right)$, and $\phi_{\mathbf{k}}$ such that $e^{-i k_{3}} \delta_{k_{1}} \delta_{k_{2}} \equiv\left|\delta_{k_{1}}\right|\left|\delta_{k_{2}}\right| e^{i \phi_{\mathbf{k}}}$. The above Hamiltonian is easily diagonalized and we obtain the spectrum to be

$$
E(\mathbf{k})= \pm \frac{1}{2 \sqrt{2}}\left[\Delta_{\mathbf{k}} \pm\left[\Delta_{\mathbf{k}}^{2}-\left\{\left(J_{z}^{2}-\left|\delta_{k_{1}}\right|\left|\delta_{k_{2}}\right|\right)^{2}+2 J_{z}^{2}\left(1-\cos \phi_{\mathbf{k}}\right)\left|\delta_{k_{1}}\right|\left|\delta_{k_{2}}\right|\right\}\right]^{\frac{1}{2}}\right]^{\frac{1}{2}} .
$$

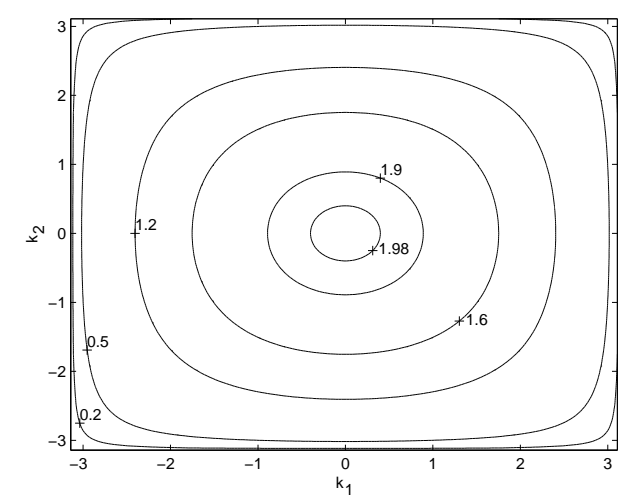

FIG. 3: In the $\mathbf{k}$ space, the contour on which the gap vanishes is projected on $k_{3}=0$ plane. $J_{x}=J_{y}=1$, and $J_{z}$ is varied between zero and two. Corresponding values of $J_{z}$ are shown next to the contours.

In the ground state, all the negative-energy states are filled. The system is gapless if solution exists for $E=0$. Since the two terms inside the curly brackets in Eq. (23) are positive definite, for $E=0$, both of them have to vanish, i.e.,

$$
\begin{aligned}
J_{z}^{2}= & {\left[J_{x}^{2}+J_{y}^{2}+2 \cos k_{1} J_{x} J_{y}\right]^{\frac{1}{2}} } \\
& \times\left[J_{x}^{2}+J_{y}^{2}+2 \cos k_{2} J_{x} J_{y}\right]^{\frac{1}{2}}, \\
\cos \phi_{\mathbf{k}}= & 1 .
\end{aligned}
$$

The values of $k_{1}$ and $k_{2}$ for which the gap vanishes are determined by Eq. (24); $k_{3}$ is then given by Eq. (25). Solutions of Eq. (24) exist only when $J_{z} \leq J_{x}+J_{y}$, $J_{x} \leq J_{y}+J_{z}$ and $J_{y} \leq J_{z}+J_{x}$; these conditions are same as that for the 2D Kitaev model. Figure 3 shows the plot of contours satisfying Eq. (24) projected on to $k_{3}=0$ plane, where we have set $J_{x}=J_{y}=1$ and varied $J_{z}$ from zero to two. The contour shrinks to the point $(0,0)$ as $J_{z}$ approaches $J_{x}+J_{y}=2$, i.e., when the gap opens up.

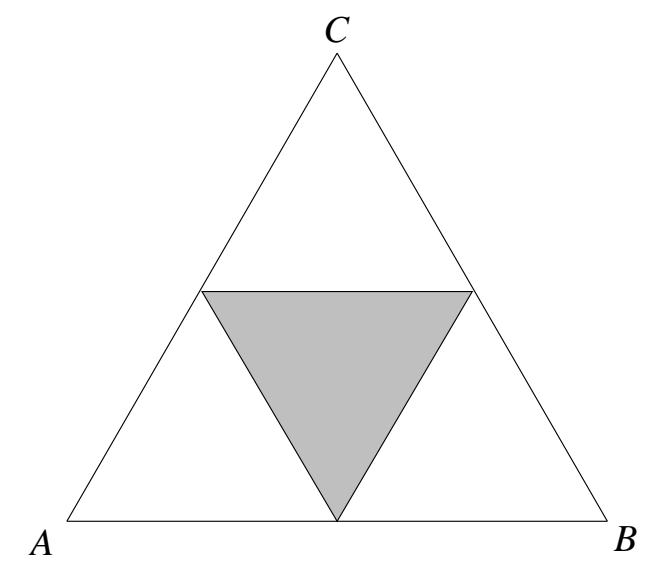

FIG. 4: The phase diagram: it shows the plane defined by $J_{x}+J_{y}+J_{z}=1$. Point $A$ corresponds to $J_{x}=1$ and $J_{y}=J_{z}=0, B$ corresponds to $J_{y}=1$ and $J_{z}=J_{x}=0$, and $C$ corresponds to $J_{z}=1$ and $J_{x}=J_{y}=0$. The shaded region is the gapless phase.

Figure 4 depicts the phase diagram on a section of the parameter phase. Interestingly, the phase diagram is symmetric in the three coupling constants though they do not appear symmetrically in the Hamiltonian (the $z$-links cannot be transformed to $x$ or $y$ links by any symmetry transformation of the lattice). Nor is the spectrum, given by Eq. (23), symmetric in the coupling constants.

We end the discussion on the ground state by noting that spin correlations can be calculated in exactly the same way as in $2 \mathrm{D} \stackrel{19,20}{=}$ The only non-zero correlations are those constructed out of the interaction terms that appear in the Hamiltonian.

\section{FLUX EXCITATIONS}

The Kitaev model in 2D has anyonic excitations, i.e., there are particle-like excitations which obey nontrivial statistics. Anyons are very specific to $2 \mathrm{D}$ and cannot exist in higher dimensions; the fundamental reason being that in $D>2$ there are no nontrivial paths which take 
a particle around another. However, in 3D, excitations localized on loops may obey nontrivial statistics. In this section, we show that there are excitations in our model which are localized on loops. However, we do not address the issue of their statistics in this paper.

We have seen that in the ground state $W_{p}=1$ for all plaquettes $p$. It then follows that the excitations are of two types: (1) Flux configurations which violate the condition $W_{p}=1$, i.e., $W_{p}=-1$ for some of the loops. It can be looked upon as creating a $\pi$ flux over those loops. (2) Fermionic excitations in the background of static configurations of $W_{p}$.

We will next show that the excitations of the first type have the structure of loops. Earlier we mentioned that not all $W_{p}$ are independent; now we will find the constraints among them. There are four types of plaquettes which are not related by translation. Let $a, b, c$, and $d$ be the labels for the different types [see Figs. [5](a)[5(d)]. Consider a part of the lattice, shown in Fig. 55(e), which consists of four adjacent plaquettes - each a different type. Here the links are labeled 1-20. Let the corresponding loop operators be $W_{a}, W_{b}, W_{c}$, and $W_{d}$, respectively. In terms of the spin variables these operators are

$$
\begin{aligned}
& W_{a}=\sigma_{11}^{x} \sigma_{12}^{z} \sigma_{13}^{y} \sigma_{14}^{x} \sigma_{17}^{x} \sigma_{18}^{x} \sigma_{8}^{z} \sigma_{9}^{y} \sigma_{10}^{x} \sigma_{1}^{x}, \\
& W_{b}=\sigma_{14}^{z} \sigma_{15}^{y} \sigma_{16}^{x} \sigma_{4}^{x} \sigma_{5}^{x} \sigma_{6}^{z} \sigma_{7}^{y} \sigma_{8}^{x} \sigma_{18}^{x} \sigma_{17}^{x}, \\
& W_{c}=\sigma_{1}^{z} \sigma_{2}^{y} \sigma_{3}^{y} \sigma_{4}^{y} \sigma_{5}^{x} \sigma_{6}^{z} \sigma_{7}^{y} \sigma_{8}^{y} \sigma_{9}^{y} \sigma_{10}^{x} \\
& W_{d}=\sigma_{11}^{x} \sigma_{12}^{z} \sigma_{13}^{y} \sigma_{14}^{y} \sigma_{15}^{y} \sigma_{16}^{x} \sigma_{4}^{z} \sigma_{3}^{y} \sigma_{2}^{y} \sigma_{1}^{y} .
\end{aligned}
$$

Using the relations $\sigma_{j}^{x} \sigma_{j}^{y} \sigma_{j}^{z}=i$ and $\sigma_{j}^{a 2}=1$,

$$
W_{a} W_{b} W_{c} W_{d}=1
$$

The above constraint has a graphical interpretation. Note that a loop operator can also be written as a product of the interaction terms on the links contained in the loop. In Fig. 5 the object (e), which is obtained by putting together the four loops (a)-(d), represents the left-hand side of Eq. (27). Evidently, each link in (e) is shared by two of the $W_{p}$ 's. Since the square of any interaction term is one, Eq. (27) immediately follows.

For open boundary conditions, relations such as Eq. (27) exhaust all the constraints. To find the configurations of $\left\{W_{p}\right\}$ which are consistent with the constraints, it is instructive to consider a lattice obtained by representing each plaquette by a single site. A plaquette has a step like structure, consisting of two rectangles perpendicular to the $x-y$ plane connected by another rectangle on the $x-y$ plane. Each loop can be uniquely represented by a point at the center of the rectangle on the $x-y$ plane [in Fig. [5(e)such points are marked by ellipses]. Let $\mathfrak{L}$ be

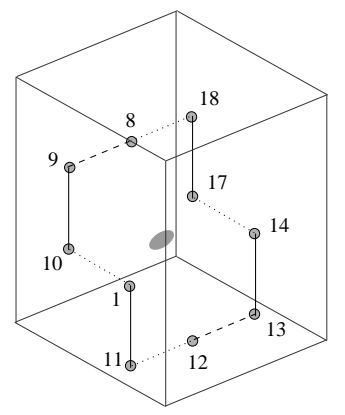

(a)

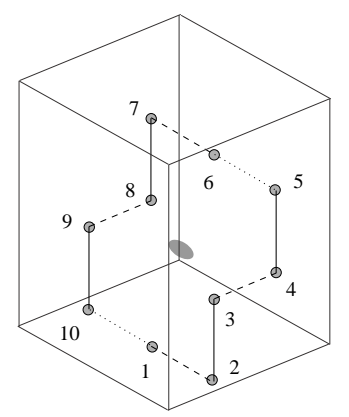

(c)

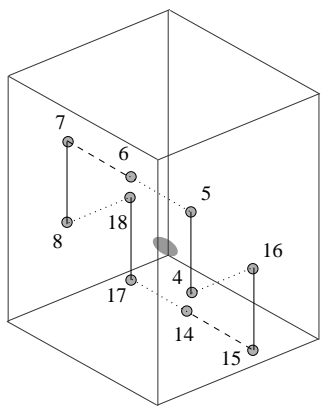

(b)



(d)



(e)

FIG. 5: [(a)-(d)] The four types of plaquettes. (e) Part of the lattice involving four such adjacent plaquettes; the corresponding operators give rise to a constraint. The ellipses, labeled $a-d$, respectively, represent each of the loops and form a "tetrahedron".

the new lattice thus obtained; topologically, it is the pyrochlore lattice, an arrangement of corner-sharing tetrahedra (see Fig. 6). (The edges of the geometrical object formed by the centers of four adjacent loops are not of equal length, and hence do not form an exact tetrahedron but a stretched one. However, the connectivity of $\mathfrak{L}$ is same as that of the pyrochlore lattice, and we will continue to refer to the basic objects as tetrahedron.)

In this description, the four plaquettes that give rise to the constraint in Eq. (27) are the four sites of a tetrahedron, and each tetrahedron corresponds to an indepen- 




FIG. 6: The lattice $\mathfrak{L}$ formed by the plaquettes - the pyrochlore lattice. Loops such as the dashed one, which goes through six sites, are the shortest (apart from the basic triangles).

dent constraint. Therefore, any configuration satisfying all the constraints will have an even number of $W_{p}$ taking value of -1 in each tetrahedron, where $p$ is now the site index in $\mathfrak{L}$. Now it is clear how to obtain such configurations: draw a loop $\mathcal{C}$ which does not cross itself and which lies entirely within the tetrahedra, and let

$$
W_{p}=\left\{\begin{aligned}
-1, & \text { if } p \in \mathcal{C} \\
1, & \text { otherwise }
\end{aligned}\right.
$$

Any closed self-avoiding loop contains an even number of sites $(0,2$, or 4$)$ belonging to any particular tetrahedron; hence all the constraints are satisfied. In other words, the flux excitations have the structure of loops in the lattice $\mathfrak{L}$.

\section{EFFECTIVE HAMILTONIAN IN THE LARGE $J_{z}$ LIMIT}

To study the excitations in the gapped phase, one can follow Kitaev's 15 approach to the $2 \mathrm{D}$ model - a perturbative analysis in the limit $J_{z} \gg J_{x}, J_{y}$. The unperturbed Hamiltonian $H_{0}$ and the perturbation $H_{1}$ are

$$
\begin{aligned}
H_{0} & =-J_{z} \sum_{<i j>z} \sigma_{i}^{z} \sigma_{j}^{z}, \\
H_{1} & =-J_{z} \sum_{<i j>_{x}} \sigma_{i}^{x} \sigma_{j}^{x}-J_{z} \sum_{<i j>_{y}} \sigma_{i}^{y} \sigma_{j}^{y}, \\
H & =H_{0}+H_{1} .
\end{aligned}
$$



(2a)


(2b)

FIG. 7: Representation of the flux operators in $H_{\text {eff }}$ associated with the four types of plaquettes.

The ground state of $H_{0}$ is highly degenerate: the two spins on each of the $z$-links can be either $|\uparrow \uparrow\rangle$ or $\mid \downarrow \downarrow$ \rangle . This degeneracy is lifted only at the sixth order of perturbation theory. We now calculate the effective lowenergy Hamiltonian to this order.

The groundstate subspace of $H_{0}$ can be thought of as the Hilbert space of effective spin $\frac{1}{2}$ 's located at each $z$ link. Let $\tau_{m}^{x}, \tau_{m}^{y}$ and $\tau_{m}^{z}$ be Pauli matrices acting on the effective spin at the $z$ link denoted by $m$, such that,

$$
\begin{aligned}
\tau_{m}^{z}|\uparrow \uparrow\rangle_{m} & =|\uparrow \uparrow\rangle_{m}, \\
\tau_{m}^{z}|\downarrow \downarrow\rangle_{m} & =-|\downarrow \downarrow\rangle_{m} .
\end{aligned}
$$

A plaquette consists of four $z$-links and six $x$ or $y$ links. Consider a plaquette by also including the two $z$-links that are directed out of the loop (see Fig. 7). Let us label the $z$-links in such an object with an index $i=1,2, \ldots, 6$. The labeling scheme for the four different types of loops is shown in the figure. We associate with each plaquette $p$ a flux operator $B_{p}$ defined as follows.

$$
B_{p}=\tau_{p 1}^{z} \tau_{p 2}^{a_{2}} \tau_{p 3}^{a_{3}} \tau_{p 4}^{z} \tau_{p 5}^{a_{5}} \tau_{p 6}^{a_{6}},
$$

where, for $i=2,3,5,6, a_{i}=x$ if the two links in the loop attached to the $i$ th $z$ link are the same; and $a_{i}=y$ otherwise. The flux operator associated with the various types of loops are also shown in Fig. 7 Then, to sixth order - which is the leading order - the effective Hamiltonian, ignoring the constant terms arising at the lower 
orders, is

$$
H_{\mathrm{eff}}=-\frac{7 J_{x}^{4} J_{y}^{2}}{256 J_{z}^{5}} \sum_{p} B_{p}-\frac{7 J_{y}^{4} J_{x}^{2}}{256 J_{z}^{5}} \sum_{p}^{\prime} B_{p},
$$

where the unprimed sum is over the loops of types $1 a$ and $2 a$ in Fig. 7 which contain four $x$ links and two $y$ links, while the primed sum is over loops of types $1 b$ and $2 b$, which contain four $y$ links and two $x$ links. Furthermore,

$$
\begin{aligned}
{\left[B_{p}, B_{p^{\prime}}\right] } & =0 \\
B_{p}^{2} & =1, \forall p, p^{\prime} .
\end{aligned}
$$

Thus, the operators $B_{p}$ can be simultaneously diagonalized for all values of $p$ and their eigenstates will also be the eigenstates of $H_{\text {eff }}$. From Eq. (35) it follows that the eigenvalues of $B_{p}$ are \pm 1 . Therefore, the ground state $|G S\rangle_{\text {eff }}$ will be such that

$$
B_{p}|G S\rangle_{\mathrm{eff}}=|G S\rangle_{\mathrm{eff}}, \quad \forall p .
$$

It is straightforward to see that $B_{p}$ is the projection of $W_{p}$ - the conserved quantities of the spin model defined in Eq. (8) - on the ground-state subspace of $H_{0}$. Therefore, in the large $J_{z}$ limit, Eq. (36) is a confirmation of our numerically verified assumption earlier that the ground state of $H$ is vortex free.

The excitations are the states which violate Eq. (36). i.e., $B_{p}=-1$ for $p$ in some subset of plaquettes. However, not all $B_{p}$ are independent - there exist constraints similar to Eq. (27) - therefore the plaquettes with $B_{p}=-1$ cannot be chosen arbitrarily. To understand the properties of the excitations, such as their statistics, a detailed analysis is required. This is currently being done.

\section{SUMMARY AND DISCUSSION}

We have constructed and solved a three-dimensional spin- $\frac{1}{2}$ model which is a generalization of the Kitaev model on a honeycomb lattice. Based on the methods used by Kitaev $\stackrel{15}{\rightleftharpoons}$ we calculated the exact low-energy fermionic spectrum by mapping the spin model to one of free fermions in the background of a static $\mathbb{Z}_{2}$ gauge field; the system has a gapped phase and a gapless one. Quite interestingly, the gap vanishes on a contour in the $\mathbf{k}$ space; this could be related to some accidental degeneracies - unrelated to any symmetry of the Hamiltonian - of the ground state in the classical limit. The two-dimensional Kitaev model has been shown to have such a degeneracy in the classical limit, which grows exponentially with the system size $\stackrel{32}{\underline{32}}$ This result can be readily generalized to our model. It will be interesting to see how quantum fluctuations lifts this degeneracy.

We have further shown that the excitations of the gauge field, due to some local constraints, have the topology of loops. As a first step towards understanding the nature of the excitations in the gapped phase, we have derived an effective $\mathbb{Z}_{2}$ gauge theory in the limit $J_{z} \gg J_{x}, J_{y}$. The ground state of the effective Hamiltonian thus obtained is trivially solved and it is consistent with the assumption we made in the calculation of the fermionic spectrum that the ground state is vortex free. Further study of the effective theory should tell us more about the properties of the excitations, such as whether they obey fractional statistics.

Yao and Kivelson 26 have introduced a two-dimensional Kitaev model in which one type of plaquettes is a triangle; the existence of loops with odd number of links results in the spontaneous breaking of time-reversal symmetry. We mention that in our model also the time reversal symmetry can be similarly broken by replacing each vertex in the lattice with a triangle.

Note added: Toward the completion of this paper, we came across the work by Si and Yu in Ref. 33 which discusses a variety of exactly solvable Kitaev models in three dimensions, none of which are identical to the model introduced here.

\section{Acknowledgments}

We thank G. Baskaran and R. Shankar for very useful discussions.
* Electronic address: saptarshi@imsc.res.in

$\dagger$ Electronic address: nsurendr@ictp.it

1 X. G. Wen, Quantum Field Theory of Many-body Systems (Oxford University Press, New York 2004).

2 R. B. Laughlin, Phys. Rev. Lett. 50, 1395 (1983).

3 X. G. Wen and Q. Niu, Phys. Rev. B 41, 9377 (1990).

${ }^{4}$ G. Moore and N. Read, Nucl. Phys. B 360, 362 (1991).

${ }^{5}$ G. Baskaran, Z. Zou, and P. W. Anderson, Solid State Commun. 63, 973 (1987).

6 X. G. Wen, F. Wilczek, and A. Zee, Phys. Rev. B 39, 11413 (1989).

7 N. Read and S. Sachdev, Phys. Rev. Lett. 66, 1773 (1991).

8 X. G. Wen, Phys. Rev. B 65, 165113 (2002).
9 D. S. Rokhsar and S. A. Kivelson, Phys. Rev. Lett. 61, 2376 (1988).

10 N. Read and B. Chakraborty, Phys. Rev. B 40, 7133 (1989).

11 R. Moessner and S. L. Sondhi, Phys. Rev. Lett. 86, 1881 (2001).

12 M. Freedman, Commun. Math. Phys. 234, 129 (2003).

13 P. Shor, Proceedings of the 35th Annual Symposium on Fundamentals of Computer Science. (IEEE Press, Los Almitos, CA, 1994), pp. 124-134.

14 A. Yu. Kitaev, Ann. Phys. (N.Y.) 303, 2 (2003).

15 A. Yu. Kitaev, Ann. Phys. (N.Y.) 321, 2 (2006).

16 C. Nayak, S. H. Simon, A. Stern, M. Freedman, and 
S. D. Sarma, Rev. Mod. Phys. 80, 1083 (2008).

17 L. M. Duan, E. Demler, and M. D. Lukin, Phys. Rev. Lett. 91, 090402 (2003).

18 A. Micheli, G. K. Brennen, and P. Zoller, Nature Phys. 2, 341 (2006).

19 G. Baskaran, S. Mandal, and R. Shankar, Phys. Rev. Lett. 98, 247201 (2007).

${ }^{20}$ H. D. Chen and Z. Nussinov, J. Phys. A: Math. Theor. 41, 075001 (2008).

${ }^{21}$ H. D. Chen and J. Hu, Phys. Rev. B 76, 193101 (2007).

22 K. P. Schmidt, S. Dusuel, and J. Vidal, Phys. Rev. Lett. 100, 057208 (2008).

23 S. Dusuel, K. P. Schmidt, and J. Vidal, Phys. Rev. Lett. 100, 177204 (2008)

${ }^{24}$ K. Sengupta, D. Sen, and S. Mondal, Phys. Rev. Lett. 100, 077204 (2008).

25 S. Yang, D. L. Zhou, and C. P. Sun, Phys. Rev. B 76,
180404(R) (2007)

${ }^{26}$ H. Yao and S. A. Kivelson, Phys. Rev. Lett. 99, 247203 (2007).

27 M. A. Levin and X. G. Wen, Phys. Rev. B 71, 045110 (2005).

28 A. Hamma, P. .Zanardi, and X. G. Wen, Phys. Rev. B 72, 035307 (2005).

29 H. Bombin, and M. A. Martin-Delgado, Phys. Rev. B 75 , 075103 (2007).

30 H. Bombin, and M. A. Martin-Delgado, Phys. Rev. Lett. 98, 160502 (2007).

31 E. H. Lieb, Phys. Rev. Lett. 73, 2158 (1994).

32 G. Baskaran, D. Sen, and R. Shankar, Phys. Rev. B 78, 115116 (2008).

33 T. Si and Y. Yu, Nucl. Phys. B 803, 428 (2008). 